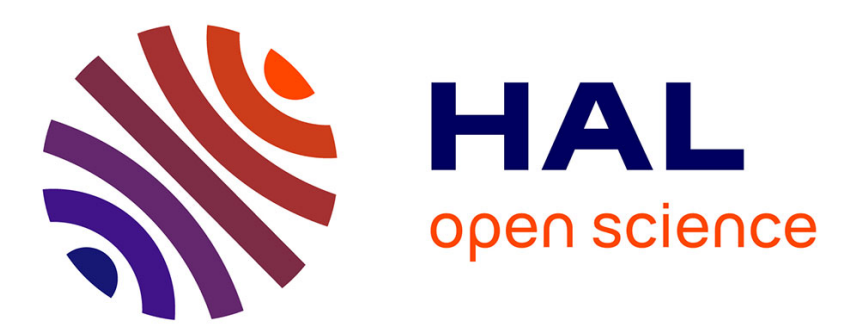

\title{
Institution scolaire et recherche biographique: prévenir la violence par la prise en compte et la mise en sens des parcours scolaires.
}

Anne Dizerbo

\section{- To cite this version:}

Anne Dizerbo. Institution scolaire et recherche biographique: prévenir la violence par la prise en compte et la mise en sens des parcours scolaires.. Les Sciences de l'éducation pour l'ère nouvelle: revue internationale, 2013, 1 (46), pp.49-71. 10.3917/lsdle.461.0049 . halshs-01158701

\section{HAL Id: halshs-01158701 https://shs.hal.science/halshs-01158701}

Submitted on 1 Jun 2015

HAL is a multi-disciplinary open access archive for the deposit and dissemination of scientific research documents, whether they are published or not. The documents may come from teaching and research institutions in France or abroad, or from public or private research centers.
L'archive ouverte pluridisciplinaire HAL, est destinée au dépôt et à la diffusion de documents scientifiques de niveau recherche, publiés ou non, émanant des établissements d'enseignement et de recherche français ou étrangers, des laboratoires publics ou privés. 


\title{
Institution scolaire et recherche biographique : prévenir la violence par la prise en compte et la mise en sens des parcours scolaires.
}

\author{
Dizerbo Anne \\ Laboratoire EXPERICE. Paris13/Nord \\ 18 rue du Dr Calmette \\ 22000 Saint-Brieuc \\ anne.dizerbo@sfr.fr
}

RÉSUMÉ. Cette contribution présente un dispositif de recherche prenant source dans des questions liées à ma pratique professionnelle d'enseignante. Il met au travail les apports de la recherche biographique dans une institution éducative en questionnement pour découvrir si le récit de leur vécu scolaire est une médiation permettant à des jeunes considérés en situation de difficulté de configurer une figure d'élève qui forme un compromis acceptable, donc le moins violent possible, entre leurs différentes identifications. Il observe si le processus de biographisation, par la reconnaissance des singularités de ses membres aussi bien que de leurs trajectoires communes, favorise la constitution d'un groupe en communauté de travail et s'il peut contribuer, par la construction du sens de leur parcours, à modifier le rapport au savoir et la posture face aux apprentissages de certains jeunes en souffrance dans une situation scolaire qui les objective.

MOTS-CLÉS : Violence. Parcours. Expérience. Biographisation. Accompagnement.

SUMMARY. This paper presents a research method inspired by issues related to my professionnal pratice as teacher. Basing on biogaphical research concepts, students facing difficulties are interviewed to find out if the narration of their school experience is a mediation that help them to bild an acceptable "student's figure", the least violent for instance. The paper examines whether the biographisation process promotes the developpement of a group to a working community and if it can contribute to the improvement of their relationship to the knowledge and learning through the acknowledgement of the singularity of its members as well as their common paths in life.

KEYWORDS : Violence. Eoute. Experience. Biographisation. Accompaniment.

RESUMEN. Este artículo presenta un dispositivo de investigación basado en cuestiones relacionadas con mi práctica profesional de docente. Conjuga los aportes de una investigación de tipo biográfico realizada en una institución educativa con el objetivo de descubrir si el relato sobre lo vivido en la escuela constituye una mediación que permite a jóvenes, considerados en situación de dificultad, configurar una figura de alumno que forme un compromiso aceptable, es decir lo menos violento posible, entre sus diferentes identificaciones. El dispositivo observa si el proceso de construcción biográfica, mediante el reconocimiento de las singularidades de sus miembros y de sus trayectorias comunes, facilita la constitución de un grupo en comunidad de trabajo y si puede contribuir, gracias a la construcción de sentido entorno a su recorrido, a modificar la relación con el saber y la postura frente a los aprendizajes de jóvenes que sufren en una situación escolar que los constituye en objeto.

PALABRAS ClAVE : Violencia. Trayecto. Biographisation. Experiencia(experimento). Acompañamiento. 


\section{Introduction}

Le ministère de l'Education nationale, devant la montée des violences scolaires, a mis en œuvre l'année dernière une série de mesures ${ }^{1}$ dans le but d'offrir à tous des chances égales et une intégration réussie. Le plus souvent répressives, elles font référence à une violence dont la source apparaît comme extérieure à l'institution. Il s'agit pour elle à la fois de garantir de bonnes conditions d'apprentissage à chacun, et d'être le levier éducatif d'une résolution sociale de cette violence.

Sans remettre en cause l'intrication et les interactions du monde de l'école et de la société, je propose ici d'observer le phénomène de violence scolaire en considérant l'institution comme un des espaces de sa production. La recherche présentée prend appui sur ce constat et questionne des jeunes de $4^{\text {ème }}$ quant au vécu de leur parcours scolaire afin de leur permettre de se positionner comme sujets de ce parcours. Cela passe par la transformation d'une figure d'élève négative, témoignant de la violence subie autant que de celle qu'elle engendre, en une figure plus positive.

Il est important dans un premier temps de présenter le contexte de production d'une violence institutionnelle dans lequel se positionne ma recherche. J'exposerai ensuite sa genèse et ses fondements théoriques avant de présenter plus en détails le dispositif mis en œuvre. Enfin, je rendrai compte des constats, effets et résultats auxquels je parviens à ce jour.

\section{L'institution scolaire productrice de violence}

Une lecture multiréférentielle de la violence scolaire me semble à même d'en faire une analyse permettant de la prendre en compte dans sa complexité et ses multiples dimensions. Nous convoquerons des auteurs dont les épistémologies (approches psychanalytique, psychologique, philosophique, socio-anthropologique, sociologique) et le niveau d'observation (de l'individu au macrosocial, en passant par l'organisationnel, le relationnel, l'institutionnel) varient mais convergent finalement pour démontrer que l'école produit elle-même une part de cette violence.

Develay $^{2}$, dès 1996, s'intéressait à la «crise» de l'école qu'il définissait comme n'étant pas uniquement conjoncturelle et mettait en avant le " divorce » entre les valeurs républicaines du système scolaire et les valeurs individualistes de la société. Cette crise intervient après une période pendant laquelle la massification scolaire et la diversification des voies de formation ont, selon Bloch ${ }^{3}$ permis d'accomplir des progrès démocratiques et de hisser le niveau de formation des Français. Dans un récent ouvrage, s'appuyant sur le résultat de plusieurs études internationales il fait un état des lieux du système éducatif français : celui-ci cumule le moins d'élèves en situation de réussite et le plus d'élèves en difficulté. D'après lui, le système se trouve aujourd'hui en échec dans sa mission de dispenser une formation démocratique, atomisant les élèves en fonction d'appartenance à des catégories socioprofessionnelles précises, ne donnant pas accès à tous à des études universitaires et offrant rarement une possibilité d'ascension sociale et économique. Cela malgré les mesures prises ces dernières décennies qui n'ont pas permis d'améliorer les résultats de ceux qui rencontrent des difficultés dès le primaire et sortent le plus tôt du système scolaire. L'équipe $\mathrm{ESCOL}^{4}$ montre d'ailleurs que les pratiques en maternelle et au primaire ont plutôt tendance à accroître involontairement les inégalités scolaires. Nous serions donc arrivés à la limite de ce que peut produire de positif la diversification des voies de formation.

Cet échec fait violence aux enfants en qui il s'incarne. Dans une société devenue incapable d'anticiper les changements très rapides qu'elle vit, dans un contexte social où « le sens des actions et

\footnotetext{
1 http://www.education.gouv.fr/cid2765/la-prevention-et-la-lutte-contre-la-violence.html

2 DEVELAY M. Donner du sens à l'école. Paris : ESF, 1996.

${ }^{3}$ BLOCH D. Ecole et démocratie. Pour remettre en route l'ascenseur économique et social. Grenoble : PUG, 2010.

${ }^{4}$ BAUTIER, E. (Dir.). Apprendre à l'école. Apprendre de l'école. Des risques de construction d'inégalités dès la maternelle. Lyon: Chroniques sociales, 2008.
} 
des identités est moins donné aux acteurs comme un allant de soi, qu'il n'est construit par eux » 5 , chacun porte la responsabilité de se réaliser et de trouver sa place par lui-même, se trouvant parfois dans un sentiment d'incompétence très culpabilisant.

En effet, l'homme, désormais moins déterminé par la société, expérimente l' «individualisme sociétal ${ }^{6}$ pour se réaliser en s'ancrant dans des milieux sociaux pluriels. La sommation institutionnelle tend à le responsabiliser y compris dans les obstacles et les échecs qu'il rencontre. Et elle s'applique aussi aux élèves. Dans cette situation, les pratiques scolaires de l'école républicaine dont la finalité était avant tout celui de la construction d'une identité nationale qui s'est élaborée sur des valeurs reconnues comme légitimes par le plus grand nombre ne sont plus efficaces.

L'école devenue à l'instar du monde du travail (par sa précarisation et le chômage de masse) un lieu de concurrence et de sélection continue (celle-ci s'opérant précédemment avant la scolarisation massive par la condition sociale et s'effectuant désormais tout au long du cursus, en fonction des résultats obtenus) ne peut conjuguer toutes ses missions : contribuer au développement de chaque individu, enseigner un savoir commun permettant à chaque jeune de s'insérer dans une nation conçue comme une collectivité et, enfin, certifier des connaissances acquises. Les enfants qui doivent y opérer des transactions entre les dimensions identitaires plurielles qui les constituent, y font l'expérience d'un conflit entre leur affirmation et leur identification aux représentations institutionnelles. Cela amène à se poser la question de ce qui leur est demandé aujourd'hui par l'institution et de ce qu'ils peuvent faire de cette demande confuse et contradictoire. Même les verbes utilisés par le ministère quand il redéfinit la mission des enseignants 7 : « former », « instruire », « éduquer », « enseigner », dont l'usage est communément synonymique viennent en réalité rendre confus les tâches et les rôles si on s'intéresse à leur étymologie contradictoire. "L'idée de formation brouille également les distinctions conceptuelles et obscurcit le discours pédagogique en s'insinuant quelque part entre "instruction", "éducation", "enseignement", "apprentissage", sans qu'on puisse lui assigner un site, la fixer sur un territoire. », précise Fabre ${ }^{8}$.

Ces contradictions, transmises à l'élève par les acteurs du système éducatif, «brouillent» sa compréhension des attentes en conformité avec lesquelles il est attendu qu'il construise son identité d'élève.

Le rôle officiel de l'élève est d'apprendre, d'acquérir de l'autonomie au fil de sa scolarité et de se former à la vie de citoyen ainsi qu'à un futur métier par sa capacité à exprimer ses opinions et son jugement. Mais à cette position objective qui n'offre pas souvent à l'enfant de continuité avec son histoire et son environnement en correspond une subjective qui détermine la façon dont il vit singulièrement ce rôle et son appartenance au monde de l'école en fonction de son histoire et des relations qu'il entretient, du sens qu'il peut attribuer à sa scolarité.

La question du sens des apprentissages, que Develay ${ }^{9}$ invite à se poser pour résoudre la crise qui se situe dans une mutation conjointe de l'école et de la société, devient ici essentielle. Les travaux de Delory-Momberger ${ }^{10}$ ont montré que le sens ne s'impose pas de l'extérieur et que le rapport au savoir relève de la construction biographique globale des enfants qui doivent en entrant à l'école configurer un nouveau monde intégrateur des identifications antérieures. A partir de connaissances qu'ils ont

${ }^{5}$ DUBET F. Ecole : la question du sens. In : J.-C RUANO-BORBALAN. (dir.) Eduquer et former. Les connaissances et les débats en éducation et en formation. Auxerre : Sciences Humaines, 1998, p.405.

${ }^{6}$ DELORY-MOMBERGER C. La Condition biographique. Paris : Téraèdre, 2009

${ }^{7}$ Ministère de l'éducation nationale. (2009). Mission du professeur enseignant en collège, au lycée général et technologique ou en lycée professionnel. Consulté le 12 mars 2011 sur EDUSCOL. http://eduscol.education.fr/cid48005/mission-duprofesseur-exercant-en-college-en-lycee-d-enseignement-general-et-technologique-ou-en-lycee-professionnel.html

8 FABRE M. Penser la formation. Paris: PUF, 1994.

9 DEVELAY M. Donner du sens à l'école. Paris : ESF, 1996.

10 DOLORY-MOMBERGER C. «Biographie, socialisation, formation », L'orientation scolaire et professionnelle [En ligne], $33 / 4 \mid 2004$, document 4, mis en ligne le 28 septembre 2009, Consulté le 15 août 2011. URL :

http://osp.revues.org/index $251 . h t m l$ 
déjà, ils peuvent en produire de nouvelles pour se penser dans une relation avec le monde et les autres, à condition d'y trouver du sens et d'y accorder de la valeur. Or ce sens et cette valeur sont tributaires de leur monde-de-vie: " ...ce monde dans lequel ils vivent et dont ils ont incorporé les habitus, les valeurs symboliques, comme la structure d'expérience et de connaissance qui leur permet d'interpréter les événements et les situations auxquels ils sont confrontés et d'appréhender ce qui leur est inconnu ${ }^{11}$. Les changements ne sont pas donc forcément acceptables pour tous dans la même mesure, rappelle Hatchuel ${ }^{12}$. Le rapport que l'enfant entretient au savoir dépend des stratégies d'attente que développe ou pas sa famille, de la manière dont elle vit le rapport à la culture, de son positionnement en tant que consommatrice, productrice ou inventrice de savoirs. Enfin le rapport au savoir dans la famille a souvent à voir avec le rapport au savoir du groupe social auquel elle s'identifie.

Si l'enfant n'est pas déterminé ou surdéterminé par ce qu'il vit dans sa famille, il s'y identifie néanmoins et l'entrée dans l'école peut lui faire violence en l'obligeant à se décentrer, et même parfois à se désancrer. Dewey ${ }^{13}$ montre que la discontinuité subie entre les expériences réalisées à l'extérieur du monde de l'école et à l'intérieur l'oblige parfois à adopter une posture défensive par rapport au savoir pour manifester son refus de la condition d'élève ou durer dans le système scolaire.

Lapassade ${ }^{14}$ explique que la ressource qu'est la dissociation pour résister à l'éclatement du moi entre ses différentes appartenances n'est tolérée à l'école que dans ses manifestations mineures. A la professionnalisation croissante de l'élève devenu «apprenant» décrite par Perrenoud ${ }^{15}$ fait face une professionnalisation de la déviance produite par l'institution scolaire et pouvant prendre différentes formes : absentéisme, refus de travail, violences physiques et/ou verbales, qui marginalisent un nombre toujours plus important d'élèves n'intégrant pas les codes scolaires. Elles témoignent en même temps de la détresse et de la résistance des enfants : «Avec la mise en scène de leur rituel, les jeunes tiennent tête aux attentes d'intégration de la société. ", écrit Wulf ${ }^{16}$. Rappelons, en nous appuyant sur ses travaux, que la construction d'une figure d'élève passe par des apprentissages de la ritualisation scolaire relevant d'une mimésis sociale et entrant en conflit avec d'autres ritualisations, celles des groupes auxquels l'enfant appartient. La définition du "métier » d'élève s'élabore donc collectivement entre déviance et normalisation.

La ritualisation scolaire, non négociable par les élèves et donc difficile à intégrer, ne fait plus consensus et entre en tension avec celle du groupe de pairs auquel l'enfant appartient. L'école par sa rigidité pousse elle-même à la construction d'une figure d'élève négative qui répond à une représentation figée de l'enfant dans un statut d' " élèves en difficulté », lui attribuant par généralisation un handicap socioculturel et le pensant comme un objet lacunaire ${ }^{17}$. Or si l'élève occupe bien une position sociale, son expérience scolaire est le fait d'un sujet. Il est difficile de lutter contre l'idéologie sous-jacente du don et l'inclination des différents acteurs scolaires devant une forme de fatalité aliénante sans le prendre en compte.

Il faut admettre qu'une part de la violence scolaire est générée par l'institution elle-même et qu'elle traverse tous les élèves. Reuter ${ }^{18}$ a démontré que certains d'entre eux répondent au conflit entre leur espace de socialisation par une adhésion totale aux normes scolaires se traduisant par l'adoption des rituels prescrits. Leur attitude, valorisée, nourrit leur estime d'eux-mêmes et leurs résultats les confortent dans leur stratégie. D'autres fonctionnent dans une dualité entre les normes scolaires et les normes du groupe de référence auquel ils appartiennent sans que cela ne menace globalement leur scolarité. D'autres enfin manifestent clairement leur rejet de la culture scolaire pour

11 DELORY-MOMBRGER C. Biographie et éducation. Figures de l'individu-projet. Paris : Anthropos, 2003.

12 HATCHUEL F. Savoir, apprendre, transmettre. Une approche psychanalytique du savoir. Paris: La Découverte, 2005.

13 DEWEY J. Démocratie et éducation. Suivi de Expérience et éducation. Paris : Armand Colin, 2011.

${ }^{14}$ LAPASSADE, G. (1998). Microsociologie de la vie scolaire. Paris : Anthropos.

15 PERRENOUD P. (2005, avril 1er). Le Métier d'élève leur appartient. Le travail scolaire entre prescription et activité réelle de l'élève. Educateur., 2005, n 4 , pp. 26-30.

16 WULF C. Anthropologie de l'éducation. Paris: L'Harmattan., 1999, p. 127

17 CHARLOT B. Du rapport au savoir. Paris: Anthropos, 2005.

18 REUTER Y. (Dir.). Une Ecole Freinet. Fonctionnements et effets d'une pédagogie alternative en milieu populaire. Paris : L'Harmattan, 2007. 
préserver leur culture d'origine ou parce que leur rapport immédiat au monde qui passe par l'agir ne leur permet pas d'accéder à l'abstraction qui fait le fond et la forme scolaire. Ils actualisent alors leur rejet dans une attitude de dévalorisation systématique des normes scolaires : usage de la force, de menaces, d'insultes, dérision contre le comportement des enseignants et des élèves conformistes...

Tous les élèves développent des stratégies pour résister à la violence de la scolarisation mais mon attention va particulièrement ici vers ceux qui sont le plus stigmatisés par des prises en charge défectologiques ou de réparation, par là-même souvent exclus du collectif de travail, et qui ne peuvent bénéficier de la reconnaissance des pairs dont ils ont besoin pour s'intégrer dans le groupe et progresser individuellement. Ce sont eux qui le plus souvent interpellent l'institution par leurs manifestations de violence, qui seront affectés dans des filières dévalorisées socialement et intérieurement, et qui, au final, quitteront tôt le système scolaire avec un sentiment d'injustice alimentant pour une part la violence urbaine considérée au départ comme responsable de la violence scolaire.

\section{Genèse et appuis théoriques}

C'est dans ce contexte que ma pratique d'enseignante de français et de professeur principal dans un collège de ZEP19 est venue me questionner. La question de l'orientation de mes élèves à la fin de la 4ème ou de la 3ème me préoccupait. Leur manque d'implication dans cette démarche cruciale les engageant dans la durée, les modalités d'affectation, les résultats souvent subis m'interpellaient. J'avais besoin d'un étayage théorique pour apporter des réponses à mes questionnements pédagogiques, didactiques et éthiques et me positionner clairement.

Je me suis donc inscrite dans la lignée des praticiens-chercheurs avec la double-visée d'enrichir les analyses de la situation scolaire et d'accompagner les participants dans la subjectivation de leur parcours scolaire. M'appuyant sur la théorie de l'inachèvement ${ }^{20}$ de l'homme, les théories de l'expérience de la Bildung et de Dewey ${ }^{21}$, et rejoignant Meirieu (2007) ou Fabre (1994) sur le plan éthique, j'ai situé globalement ma relation aux élèves dans un partenariat tant dans le cadre de mes cours que dans celui de ma recherche, adoptant le principe de retenue de Michel Serres proposant de disposer d'un pouvoir sans en faire usage pour conjuguer éducabilité et non réciprocité : "le crédit sans la dette ».

Il me paraissait intéressant de penser les difficultés identitaires des élèves avec les apports de la recherche biographique ${ }^{22}$ afin de prévenir et remédier à la violence d'un parcours scolaire souvent vécu comme une assignation à obtenir une qualification. La recherche biographique s'attache à aborder les questions de narrativité, de réflexivité de l'écriture et du sujet ainsi que celles des temporalités afin de faire émerger: "le présent agissant, le passé comme expérience et l'avenir comme horizon de responsabilité $»^{23}$. Elle s'intéresse à l'ensemble des activités par lesquelles l'être humain est capable de se représenter l'inscription de son existence dans le temps et s'appuie entre autres sur les productions de discours narratifs en s'intéressant à leur fonction performative. L'individu, dissocié, dont la vie repose sur des crises, les dépasse en disposant de matériaux narratifs propres à lui permettre de se raconter dans différents groupes. Faisant appel dans son travail de biographisation permanent à des modèles narratifs qui rappellent le roman de formation, il met ses expériences en sens, en cohérence, dans une logique d'expérience et d'apprentissage. Ainsi la biographisation, espace de conciliation entre différentes identifications, me semblait apte à aider l'enfant à construire une figure d'élève compatible avec son monde de vie.

\footnotetext{
19 Zone d'éducation prioritaire.

${ }^{20}$ LAPASSADE G. L'Entrée dans la vie. Essai sur l'inachèvement de l'homme. Paris : Anthropos, 1997.

${ }^{21}$ DEWEY J. Démocratie et éducation. Suivi de Expérience et éducation. Paris : Armand Colin, 2001

22 DELORY-MOMBERGER C. \& CLEMENTINO DE SOUZA E. Parcours de vie, apprentissage biographique et formation. Paris : Téraèdre, 2009.

23 BACHELARD D. \& PINEAU G. (Coord.) Le biographique, la réflexivité et les temporalités. Articuler langues cultures et formation. Paris : L'Harmattan, 2009
} 
Il s'agissait d'observer si un travail sur le récit du parcours scolaire, souvent considéré comme un passif, était pour l'élève le moyen d'y porter un nouveau regard distancié et de le mettre en sens. Cette reconfiguration, soumise au regard rassurant des pairs et préservant une continuité identitaire, permettait d'accorder au parcours de la valeur et de s'en accorder à soi-même, suffisamment pour devenir sujet d'un projet d'orientation impliquant la construction d'une nouvelle figure d'élève et un changement de posture face aux apprentissages.

Le travail envisagé avec les élèves, prenant en compte leur singularité dans leurs multiples dimensions et tensions avec le contexte et les groupes dans lesquels ils évoluent, était susceptible de leur permettre de transformer l'espace de concurrence du groupe classe en communauté de travail où s'engager en confiance dans une spirale de réussite favorable à un changement de posture face aux apprentissages. Ne se centrant sur aucun des pôles du système éducatif : enseignant, apprenant, ou savoir, mais plutôt sur les rapports qu'ils entretiennent entre eux et avec l'environnement, il observait le processus identitaire en œuvre dans la construction d'une figure d'élève (qui ne se constitue pas indépendamment d'une personne concernée par la finalité émancipatrice de l'acte éducatif).

\section{Le dispositif}

Pour accéder à une compréhension herméneutique du processus de construction identitaire à l'œuvre dans la narration et prendre en compte les élèves dans leurs interactions, $j$ 'ai fait le choix d'une démarche empirique et qualitative qui s'inscrit à la fois dans le champ de la recherche pédagogique et dans celui de la recherche biographique. Ma recherche repose sur le recueil d'entretiens, plus ou moins directifs suivant les étapes du travail. Le but était d'enrichir les analyses de la situation scolaire et d'accompagner les participants, mes élèves, dans la subjectivation de leur parcours en accédant à une compréhension de leur discours narratif, à leur façon de se biographier et au sens qu'ils donnent à l'école. Dans la mesure où j'avais le souci de mettre en œuvre une approche compréhensive des conduites des élèves et de placer mon écoute non sur un objet d'étude mais sur des sujets dont je recueillais des données biographiques et que j'accompagnais dans un travail éducatif, j'ai adopté une posture d'accompagnement clinique ${ }^{24}$.

Cette posture s'est déclinée différemment selon que nous étions en entretiens collectifs ou en entretiens individuels. Une réflexion théorique a été nécessaire pour répondre à l'exigence éthique que soulevait ma sur-implication auprès des élèves dans le cadre du dispositif proposé. Je me suis en effet trouvée en dehors d'une relation pédagogique ordinaire. Sortant du cadre strict de mes obligations professionnelles, j'ai quitté mes postures éducatives contractuelles ou d'autorité telles qu'elles sont définies par Le Bouëdec ${ }^{25}$ pour adopter dans les moments d'entretiens collectifs une posture d'animatrice en ce sens que je me suis mise au service des élèves qui produisent leur récit sans participer directement mais en prenant en charge l'organisation et la régulation du groupe. Il s'agissait alors pour moi dans ce cadre de me mettre à l'écoute de la demande implicite de l'élève d'être entendu et reconnu sans perdre de vue l'objectif qui était de constituer une communauté de travail.

Mais lors des entretiens individuels, c'est une posture d'accompagnement que j'ai adoptée : j'ai écouté et accueilli les récits, aidé les élèves à discerner les points leviers de l'histoire scolaire qu'ils produisaient, et j'ai cheminé à leur côté, acceptant que leur récit me transforme aussi, cherchant et apprenant en même temps qu'eux, en collaboration avec eux.

Boutinet ${ }^{26}$ met en évidence que l'accompagnement concerne avant tout les adultes et qu'en situation scolaire il se trouve limité par la normativité pédagogique qui le ramène à une variante de préceptorat. Le dispositif que j'ai mis en œuvre oblige néanmoins à prendre en compte les aspects théoriques dégagés dans le cadre de la formation pour adulte pour mieux situer ma pratique.

La figure contemporaine de l'accompagnateur est celle d'un individu qui se met au service d'un autre pour l'accompagner dans des transformations et c'est bien l'objectif que j'ai poursuivi.

\footnotetext{
24 NIEWIADOMSKY C. Recherche biographique et clinique narrative. Toulouse : Erès, 2012, pp. 33 à 41.

25 BOUTINET J.-P. (Dir.) Penser l'accompagnement adulte. Paris : Puf, 2007 p. 175-178.

26 BOUtINET J.-P. (Dir.) Penser l'accompagnement adulte. Paris : Puf, 2007, p. 11.
} 
L'accompagnement induit une posture paradoxale à plus d'un titre puisque: «Penser l'accompagnement, c'est faire fi d'une situation hiérarchique déclarée pour se situer dans une horizontalité plus informelle aux allures paradoxales, au sein de laquelle une égalité de surface entre les personnes impliquées masque un déséquilibre de fait entre le relatif confort existentiel de l'une et celui plus problématique de l'autre $»^{27}$

L'accompagnement induit nécessairement une asymétrie de relation qui peut faire violence à l'accompagné puisqu'elle ne peut faire preuve de réciprocité. Mauss ${ }^{28}$ a parfaitement démontré à ce sujet le caractère agoniste $\mathrm{du}$ don. L'asymétrie disparaît dans la réciprocité réflexive de l'accompagnement puisque l'élève, le groupe et moi dans un premier temps, et l'élève et moi dans les entretiens individuels, trouvons dans une mutualité coopérative une parité de relation à défaut d'une parité de place, sur laquelle s'appuie une reconnaissance mutuelle. Cette relation repose en ce qui me concerne sur une forme d'écoute particulière, une « écoute sensible » au sens où l'entend Barbier29, une écoute dialogique et non silencieuse, qui engage ma responsabilité d'être humain avant celle de l'enseignante, sans toutefois l'exclure, et qui préserve l'autonomie de l'élève.

J'ai souvent craint que le recueil des entretiens ne vienne « bousculer» mes élèves en accélérant un processus dans lequel ils n'étaient peut-être pas tous près à s'engager. J'ai donc essayé d'être prudente sur la nature de mes questions et ma façon de recevoir les réponses. Je me suis d'abord fixée comme limite de ne pas aller au-delà de ce qui pouvait être utile à mon interlocuteur. Ce qui amenait une nouvelle question : comment savoir si la question posée allait faire avancer l'élève ou pas, si elle ne risquait pas de le déstabiliser ? Par ailleurs l'exercice demandait à ce que l'on se questionne sur le regard à poser et renvoyer sur les énoncés de l'élève. Pouvais-je tout entendre sans jamais donner un avis? Mes responsabilités d'enseignante et de professeur principal ne me permettaient pas de rester neutre. Il fallait néanmoins créer une certaine ouverture pour que ce travail, à la lisière de l'espace scolaire, se fasse en confiance et sans faire violence à l'élève. Tout était histoire de limites à trouver avec cette difficulté qu'elles sont invisibles extérieurement et qu'on ne peut que les «sentir » dans la situation sans écarter définitivement le risque de se tromper. J'ai du me résoudre à vivre l'incertitude, à tendre vers une forme d'écoute et d'accompagnement sans jamais pouvoir être sûre de l'atteindre en situation, à trancher dans l'instant et au cas par cas. J'ai finalement admis qu'il ne pouvait y avoir de confort dans ma posture d'accompagnement qui ne soit une menace pour la relation instaurée.

J'appuierai ici ma réflexion sur des données recueillies dans mon établissement scolaire avec deux groupes différents à un an d'intervalle.

Tableau récapitulatif des données contextuelles de la recherche

\begin{tabular}{|l|l|l|}
\hline & $\begin{array}{l}\text { Année 1 } \\
\mathbf{2 0 0 9 - 2 0 1 0}\end{array}$ & $\begin{array}{l}\text { Année 2 } \\
\mathbf{2 0 1 1 - 2 0 1 2}\end{array}$ \\
\hline Classe & Classe « expérimentale » ZEP & Classe « ordinaire » ZEP \\
\hline Volontaires & \multicolumn{1}{|c|}{$16 / 16$} & $25 / 25$ \\
\hline Profil des classes & $\begin{array}{l}\text { Faible niveau } \\
\text { Tendance à l'absentéisme, au } \\
\text { découragement, à l'agitation en classe ou } \\
\text { dans l'enceinte du collège. }\end{array}$ & $\begin{array}{l}\text { Classe ne posant pas de problème de gestion } \\
\text { particulier dans sa globalité. }\end{array}$ \\
\hline
\end{tabular}

${ }^{27}$ Idem, p. 7.

28 MAUSS M. Essai sur le don. Paris : PUF, 2007.

29 BARBIER R. L'Approche transversale. Paris : Anthropos, 1997. 


\begin{tabular}{|c|c|c|}
\hline & $\begin{array}{l}\text { Certains élèves se connaissent depuis le } \\
\text { primaire et se connaissent au moins de vue } \\
\text { Elèves en situation de grande difficulté } \\
\text { dans plusieurs matières, avec une mauvaise } \\
\text { estime d'eux-mêmes. } \\
7 \text { ont commencé leur scolarité dans un autre } \\
\text { pays ou dont les parents ne parlent pas } \\
\text { couramment le français. }\end{array}$ & $\begin{array}{l}\text { Certains élèves se connaissent depuis le } \\
\text { primaire et se connaissent au moins de vue } \\
\text { Un tiers d'élèves obtenant de très bons } \\
\text { résultats, un tiers d'élèves en très grande } \\
\text { difficulté, un tiers d'élèves avec des résultats } \\
\text { moyens. } \\
8 \text { élèves ayant entamé leur scolarité dans un } \\
\text { autre pays ou dont les parents ne parlent pas } \\
\text { couramment le Français. }\end{array}$ \\
\hline Objectifs prioritaires & $\begin{array}{l}\text { Donner ou redonner confiance en soi et } \\
\text { goût à l'école. }\end{array}$ & Classique. \\
\hline Equipe pédagogique & Enseignants volontaires en grande partie. & Fonctionnement classique \\
\hline Modalité de travail & $\begin{array}{l}\text { Emploi du temps classique avec une plage } \\
\text { de travail inter et transdisciplinaire. } \\
\text { Concertation pour construire des outils } \\
\text { méthodologiques et réflexion approfondie } \\
\text { sur la question de l'évaluation et de la } \\
\text { notation. Modalités d'évaluation non } \\
\text { chiffrée dans plusieurs disciplines. Prise en } \\
\text { compte des progrès autant que des } \\
\text { performances. }\end{array}$ & Fonctionnement classique \\
\hline $\begin{array}{l}\text { Acteurs engagés } \\
\text { dans le dispositif }\end{array}$ & $\begin{array}{l}\text { Accord du chef d'établissement. } \\
\text { Communication avec les inspecteurs } \\
\text { pédagogiques du dispositif et des bilans. } \\
\text { Accord des familles et des élèves. } \\
\text { En concertation avec la conseillère } \\
\text { principale d'éducation et la conseillère } \\
\text { d'orientation psychologue. } \\
\text { Avec la participation des collègues pendant } \\
\text { le temps de biographisation collective. }\end{array}$ & $\begin{array}{l}\text { Accord du chef d'établissement. } \\
\text { Communication avec les inspecteurs } \\
\text { pédagogiques du dispositif et des bilans. } \\
\text { Accord des familles et des élèves. } \\
\text { En concertation avec la conseillère } \\
\text { principale d'éducation et la conseillère } \\
\text { d'orientation psychologue. } \\
\text { Avec la participation des collègues pendant } \\
\text { le temps de biographisation collective. }\end{array}$ \\
\hline $\begin{array}{l}\text { Objectifs de } \\
\text { recherche }\end{array}$ & $\begin{array}{l}\text { Observer les constructions de figure d'élève } \\
\text { et les effets de la biographisation collective } \\
\text { et individuelle }\end{array}$ & $\begin{array}{l}\text { Affiner l'observation de la construction } \\
\text { d'une figure d'élève et la subjectivation du } \\
\text { parcours }\end{array}$ \\
\hline Modalités de travail & $\begin{array}{l}\text { Plage banalisée pour l'entretien collectif et } \\
\text { le temps d'écriture. Plages libres dans le } \\
\text { temps scolaire en fin de journée. }\end{array}$ & $\begin{array}{l}\text { Cours de français et heure de } \mathrm{PPRE}^{30} \text { en fin } \\
\text { de journée }\end{array}$ \\
\hline $\begin{array}{l}\text { Nombre d'élèves } \\
\text { ayant pris la parole }\end{array}$ & 15 & 23 \\
\hline $\begin{array}{l}\text { Nombre d'élèves } \\
\text { ayant produit un } \\
\text { récit écrit }\end{array}$ & 16 & 25 \\
\hline $\begin{array}{l}\text { Nombre d'élève } \\
\text { ayant accepté de } \\
\text { participer à un } \\
\text { entretien individuel }\end{array}$ & 10 & 18 \\
\hline
\end{tabular}

30 Programme personnalisé de réussite éducative 


\begin{tabular}{|l|l|l|}
\hline $\begin{array}{l}\text { Nombre d'entretiens } \\
\text { menés }\end{array}$ & 8 & 12 \\
\hline
\end{tabular}

Les volontaires étaient issus de classes de 4ème, deuxième année du cycle central réputée pour être délicate et plus difficile que les autres avec des élèves en pleine croissance qui connaissent d'importantes interrogations identitaires et à qui on demande de se questionner tôt sur leur orientation professionnelle. Ce niveau peut ouvrir sur un abandon de la filière générale avec une inscription dans un lycée professionnel pour entreprendre une 3ème DP $6^{31}$ ou entrer au CFA ${ }^{32}$. La particularité des classes avec lesquelles j'ai effectué cette recherche tenait à la difficulté des élèves à intégrer le monde scolaire dans ce qu'il comporte d'attentes de travail et de résultats, à leur tendance à se réfugier dans une conduite déviante, dans l'absentéisme, dans l'agressivité.

Après avoir obtenu leur accord et établi un contrat portant sur la confidentialité, le respect et la propriété des entretiens, j'ai interrogé les élèves sur leur parcours scolaire de l'entrée à l'école jusqu'au moment des entretiens ainsi que sur la façon dont ils envisageaient son prolongement. Ce premier travail qui avait comme finalité de constituer des communautés de travail propices à l'expression des singularités a pris forme dans un dispositif collectif. Il s'est poursuivi par un temps d'écriture individuelle du parcours exploré oralement. J'ai ensuite mené avec certains volontaires des entretiens semi-directifs afin de les accompagner, par un travail de conscientisation, dans la construction d'une historicité visant à leur permettre de s'engager en tant que sujet dans leur projet d'orientation, de construire une identité d'apprenant, de modifier leur rapport à l'école. Réécritures et relectures accompagnées du parcours scolaire étaient nécessaires pour progressivement réévaluer des expériences perçues négativement et configurer l'histoire scolaire dans une perspective émancipatrice.

Pour analyser les entretiens, j'ai bâti ma propre trame de lecture en m'appuyant sur les catégories d'analyses de Heinz adaptées par Delory-Momberger ${ }^{33}$, sur les propositions de Varro en matière de temporalités et de langage, visant à distinguer « la partie récit (constitutive de l'identité narrative de l'interviewé) du discours (l'interaction dans un temps partagé). ${ }^{34}$ J'ai ensuite affiné cette trame d'analyse biographique à partir de ma lecture de Reuter ${ }^{35}$ et de ma découverte de la méthode «NIM » ${ }^{36}$.

Je me suis inspirée de ces méthodes sans m'en tenir rigoureusement à leur protocole mais en les adaptant pour laisser pleinement la place à une interprétation herméneutique en accord avec le type d'écoute utilisé pendant les entretiens. J'ai essayé de mettre en évidence les liens entre les dyades temps et temporalités, langues et langage, récit et discours, de repérer les actants et le rapport entretenu entre eux et l'auteur-narrateur et enfin d'observer l'organisation thématique du récit et les schémas d'action qui s'en dégageaient.

Dans un premier temps, à partir de l'ensemble des récits et de toutes les autres informations en ma possession collectées pendant des conversations informelles et des réunions, ainsi que sur les bulletins scolaires, j'ai essayé d'établir une chronologie des éléments, des faits biographiques qui me permettaient de comprendre comment l'élève vit objectivement son parcours. Dans un second temps, je me suis attachée à la façon dont ces événements étaient racontés et vécus. J'ai découpé le texte en unités de sens, de thème, de discours et $\mathrm{j}$ 'ai observé la fonction performative des paroles, les indices de subjectivité et de modalisation présents, les types de verbes en fonction de leur fonction expressive (sentiment, action, jugement) et de leur voix (active, passive). Enfin, j'ai synthétisé ces deux analyses pour essayer de comprendre pourquoi les faits étaient racontés comme ils le sont.

31 DP6 : Troisième découverte professionnelle 6 heures.

32 CFA : Centre de formation pour apprentis.

33 DELORY-MOMBERGER C. Cours sur l'analyse d'entretien, 2010.

34 VARRO G. Temporalité(s) et langage dans l'analyse des entretiens biographiques, 2008. Consulté le 13 avril 2010 sur

Temporalités. Revue de sciences sociales et humaines, $n^{\circ} 8$. http://temporalites.revues.org/index123.html.

35 REUTER Y. L'analyse du récit. Paris : Armand Colin, 2005.

36 Biographic Narrative Interpretive Method : méthode d'interprétation des entretiens biographiques-narratifs 
M'appuyant sur les travaux de Reuter ${ }^{37}$, j'ai ensuite procédé à une analyse narratologique et énonciative des entretiens ainsi que des récits écrits produits par les élèves sur lesquels ils ont pu s'exprimer. Elle reposait sur le classement des types de verbes (action, état, jugement, sentiment, etc.), les indices de l'énonciation, les modalisations, les tournures (actives, passives), les champs lexicaux utilisés, l'observation des actants et de leur positionnement. Il s'agissait d'observer quel était le degré de subjectivité de l'élève narrateur. En effet, un « on » ou un « nous » généralisateurs, par exemple, ne réfèrent pas au même statut d'actant et le passage de l'un à l'autre au cours des différents récits d'un même élève peut s'avérer significatif. Un « je » à la tournure passive ou uniquement sujet de verbes exprimant des ressentis peut évoquer une objectivation du statut d'élève.

\section{Constats, effets, résultats}

Mes observations s'appuient sur les transcriptions et leurs analyses, le comportement des participants, les entretiens informels avec eux et l'équipe pédagogique ainsi que les bulletins scolaires.

C'est dans l'intimité de l'écriture que s'exprime avec le plus de clarté et de force la souffrance de certains élèves : " J'ai plus envie de travailler, je sais même pas pourquoi je viens. Enfin si, sûrement par obligation. Les profs ils font genre de bien nous aimer alors qu'ils sont juste là pour noter, coller, etc. Les profs ne nous aiment pas, ils sont souvent un stress, une angoisse pour nous", "Suivant les profs, j'ai mal au ventre avant d'entrer dans leur salle", "C'est dur pour moi l'école, mon parcours il est nul, je suis nul ».

Mais une des premières caractéristiques observée dans les entretiens collectifs est que ce qui est vécu comme un échec dans le cadre scolaire est souvent source d'angoisse, d'humiliation, de honte et engendre une souffrance qui affecte le jeune globalement mais dont il ne parle pas souvent ouvertement :

"Adrien: Ben moi, ça m'a permis de dire mon parcours scolaire parce que c'est des choses que j'avais jamais dit auparavant, donc euh... ça m'a permis de lâcher de... Je sais, quoi... Je l'ai dit.

Manon : Ça fait du bien!

Alexandre : Ben ouais.

Enquêteur : Alors dans quel sens ça t'a fait du bien de dire?

Adrien : Ben, je vais dire, alors je gardais tout ça pour moi et euh... mais je le disais à personne, alors à force euh.... ça s'accumule alors voilà, je l'ai dit, je l'ai dit, ça va mieux quand même...

Célia : Ouais, je suis d'accord avec toi...

Enquêteur: Tu es d'accord avec lui?

Célia : Oui.

Manon : T'avais un petit poids lourd dans ton cour en fait... C'est... voilà.

$[\ldots]$

Zoé : Ben je l'ai dit à personne moi avant, je l'ai dit à ma mère, mais...

Enquêteur: C'est la première fois que tu en parles, autrement?

Zoé : Oui. Ça soulage...

Enquêteur : Ça soulage?

Groupe : Ouais.

Alexandre : Ben... on l'avait enfermé dans la tête. »

${ }^{37}$ REUTER Y. L'Analyse du récit. Paris: PUF, 2009. 
Dans cet extrait, l'idée de pesanteur domine : « lâcher », « poids lourd », «s'accumule » associée à l'isolement «je le disais à personne», «enfermé», et au soulagement. L'hétérobiographisation collective a permis, dans le partage des expériences, d'accéder à une reconnaissance des pairs et de l'institution et a autorisé chacun à s'exprimer, à affirmer sa singularité tout en mettant à jour ses liens avec le groupe comme en témoigne ce passage d'une fin d'entretien individuel réalisé après-coup :

« Enquêteur: Ce travail sur le parcours scolaire qu'on a mené depuis le début. J'aimerais avoir ton avis. Comment tu t'es senti dans ce travail? Dans les différentes étapes?

Sébastien: Ben... C'est... Je sais pas trop moi. Je me sentais bien. Ben j'ai... j'ai dit tout ce que j'ai, mon parcours, c'que j'ai fait, tout. Ben sinon, ben... J'ai accepté pour parler de mon parcours déjà. Que tout le monde sache ce que je faisais et tout.

Enquêteur: C'était dirigé vers les autres élèves de la classe?

Sébastien: Ouais. Et j'ai vu que y avait pas que moi aussi qui était comme moi. Y avait d'autres élèves aussi. Comme euh...ben, c'est comme à peu près, ben y en a, à peu près comme moi, à peu prè,s mais sauf que c'est le même parcours. Je vois que y en a qui z'ont fait à peu près le même parcours que moi aussi. Donc, voilà.

Silence.

Enquêteur: C'était intéressant pour toi de découvrir ça?

Sébastien: Je me rendais pas compte que les autres c'était pareil, je savais pas qu'ils avaient pareil que... ben ce qu'ils faisaient et tout. Je savais qu'ils avaient des difficultés en cours, mais sinon ben les autres trucs, je savais pas pourquoi. On en parle pas trop. J'en avais parlé qu'à mes parents c'est tout. »

Ce type de prise de conscience a favorisé l'émergence d'un climat d'entre-aide plutôt que de concurrence. Le travail de biographisation est venu soutenir les efforts de l'équipe pour permettre de diminuer considérablement l'absentéisme et d'améliorer les rapports avec les camarades et les adultes de la classe.

La biographisation collective a aussi imposé un véritable travail de configuration en limitant les possibilités du narrateur de s'arranger trop facilement avec son histoire de part la nécessité de vraisemblance et de crédibilité du récit. Les auditeurs "demandent des comptes» lorsqu'ils rencontrent des incohérences. Occasion d'une multiplication des interprétations possibles, elle a également parfois permis à l'élève de commencer à se dégager de la sienne, d'en choisir une autre. Par exemple, dans le récit de Marion, une des élèves interviewées, apparaissent les ruses qu'elle déploie pour ne pas être blessée trop profondément par des échecs dans ses tentatives d'apprentissages, assumant plus facilement une posture déviante. Mais au fil du travail réalisé, son attitude en classe et son discours montrent qu'elle accepte progressivement le risque de l'erreur. On observe dans son récit la construction d'une projection de son parcours de formation qui prend en compte plus efficacement à la fois ses aspirations et les attentes familiales tout en s'attachant à trouver une continuité entre l'ancienne figure d'élève et celle qui s'élabore.

J'ai aussi pu noter une interaction entre les récits. Des modèles ou schèmes biographiques dont certains élèves disposaient étaient manifestement transmis à d'autres. Et j'ai pu observer que les élèves construisaient et faisaient évoluer collectivement des critères de normalité propre à leur groupe classe qui correspondait à une figure d'élève qu'ils s'appropriaient singulièrement, à l'écart des normes institutionnelles telles qu'elles étaient entendues.

Ce qui apparaît également, davantage dans les entretiens individuels, c'est qu'une grande partie des élèves entendus semblent accepter l'idée que la réussite scolaire n'est pas pour eux. Ils se trouvent confrontés à une représentation qui les précède et les dépasse, selon laquelle tout le monde ne peut pas réussir de la même manière et ils seraient du côté de ceux qui ne peuvent y arriver. Nous avons pu commencer à questionner ensemble ces représentations fatalistes. Cette interprétation peut correspondre à une interprétation familiale difficilement discutable :

«Enquêteur : Qu'est-ce qui faisait penser à ta mère que tu ne t'en sortirais pas en 5ème? 
Jean : J'sais pas. Elle me disait: "t'arriveras jamais en 5ème, ta sœur elle faisait pareil que toi". "

Il peut également refléter l'idée que le jeune se fait lui-même de son parcours et qu'il généralise :

« Maxime: Ben, si on peut pas aller en général et qu'on reste dans le professionnel, c'est.... C'est pas pareil mais après au final on passera un bac, c'est pareil. Et puis bon.

[...] Ben si on a pas, ben... comment dire euh... pour suivre les cours en général, ben, moi je pourrai pas quoi... alors que dans le professionnel, ce que je veux suivre ben ce sera plus facile que si j'entrai en général après que je suive en général. Parce que là ben c'est plus facile que une 4ème normale, après, une 3ème normale...

Enquêteur : Donc tu penses que tu ne pourrais pas suivre ailleurs...

Maxime : Oui. Déjà en 6ème normale, 5ème normale je sui ... je suivais pas trop. »

Les entretiens individuels pour leur part ont permis aux élèves d'entrer plus profondément dans un travail d'historicité sur leur parcours pour envisager l'intégration d'un projet de formation choisi à un projet de vie plus globale, modifiant pour cela leur rapport aux apprentissages et au savoir. C'est souvent l'usage des pronoms et des types de verbes qui en témoignent de façon flagrante. Certains élèves usant dans les entretiens collectifs d'un «nous », d'un « on » ou d'un « tu » généralisateur de l'expérience : "Quand on arrive on $6^{\text {ème }}$ », "Quand tu déménages », passent au fil du travail à un « je » qui exprime la singularité de son parcours. Après des tournures à la voix passive ou une expression dominante des sentiments éprouvés, les entretiens individuels permettent l'élaboration d'un discours plus engageant dont l'élève se trouve plus souvent sujet (verbes d'action, voix active), et plus distancié (usage de la modalisation, verbes de jugement). Cela s'est manifesté dans la classe expérimentale par un engagement accru des élèves dans une réflexion sur leur orientation et leur avenir ainsi qu'une participation active à des portes ouvertes. Ils ont trouvé tous les seize de façon autonome des stages en entreprise, fait rare pour ce type d'élèves.

L'analyse montre également que c'est par le travail réalisé en entretien individuel que se finalise la prise de conscience d'une inscription de son parcours dans une trajectoire sociale commune à beaucoup de jeunes. Ce fût le cas pour Adriana, angolaise en France depuis moins de deux ans au moment des entretiens, qui a poursuivi sa scolarité avec une meilleure estime d'elle-même, ne portant plus à elle seule le poids de ses échecs et retrouvant une confiance nécessaire pour accepter ses résultats provisoires sans se remettre en cause personnellement. En grande difficulté en début de $4^{\text {ème }}$, elle a depuis obtenu son $\mathrm{DNB}^{38}$ avec mention et s'est engagée en lycée professionnel dans une formation réfléchie et choisie.

Cette investigation menée pendant deux ans ouvre sur des questions, certaines s'appuyant sur les limites rencontrées dans ce travail. En effet, pour des élèves pourtant volontaires, je n'ai pas remarqué de changement d'attitude indiquant un déplacement identitaire vers la construction d'une figure d'élève plus positive. Je peux dans ce cas me demander si leur démarche était personnelle ou suivait le mouvement du groupe. Par ailleurs, lorsque le récit d'un élève évolue dans le sens d'une subjectivation de son parcours le rendant moins douloureux, son attitude change aussi dans mon cours, mais ce n'est pas nécessairement le cas dans celui de mes collègues. Cela invite à se questionner sur l'influence du regard porté par l'enseignant ou le groupe sur la situation de l'élève. On peut encore envisager qu'il lui est impossible d'évoluer sans risquer de mettre en danger la cohérence qu'il peut donner à son existence et certains font d'ailleurs le choix de ne pas participer.

On peut aussi vérifier si les capacités narratives et les compétences biographiques ne sont pas déficitaires. J'ai pu en effet noter une interaction importante entre les différents récits. Des modèles ou schèmes biographiques dont certains élèves disposaient étaient manifestement transmis à d'autres qui ne savaient comment se raconter dans un premier temps. Ce constat questionne le lien entre narration et biographisation et invite à penser la possibilité d'une éducation envisageant l'acquisition de capacités narratives propres à améliorer les compétences biographiques nécessaires à une subjectivation du parcours.

38 DNB : diplôme national du brevet 
Il sera aussi intéressant de voir si une co-construction des analyses avec le retour des élèves à l'occasion d'un entretien supplémentaire permet d'accéder à un niveau de réflexivité encore plus approfondi, en ayant le souci de ne pas les figer dans leur nouvelle configuration identitaire, d'inscrire leurs représentations dans une dynamique évolutive. Cela me permettrait aussi d'orienter le dispositif vers une déconstruction plus efficace de leur représentation du bon élève qui repose bien souvent sur l'idéologie du don et de la fatalité.

Par ailleurs j'ai pu observer que les élèves construisaient collectivement les critères de normalité propre à leur groupe classe, qu'ils les font évoluer et qu'ils s'approprient singulièrement, en marge des normes institutionnelles. Il convient donc de ne pas mettre à l'écart la dimension collective essentielle dans un travail de remédiation qui a trop souvent tendance à l'ignorer et à stigmatiser l'élève en situation de difficulté.

Il reste à signaler un autre effet de cette recherche. Ma posture de praticienne chercheuse a impliqué que je sorte de ma mission contractuelle d'enseignante pour entrer dans une relation d'accompagnement et d'entraide mutuelle avec les participants. Cette mutualité coopérative me permettait de me situer avec mes élèves dans une parité de relation, à défaut d'une parité de place. L'hétérobiographie a remis fortement en question mon identité professionnelle et mon positionnement éthique et elle m'a transformée autant qu'elle a transformé mes élèves en me permettant d'effectuer moi-même ce à quoi je les engageais : un travail sur l'historicité de mon parcours qui a entrâ̂né une modification de mon propre rapport au savoir. Elle m'a amenée à redéfinir ma fonction d'enseignante mais aussi ma représentation de l'identité d'élève. Je me suis longuement demandé si je devais ou pas adopter une posture clinique en accompagnant mes élèves à partir d'entretiens biographiques dans un travail formatif sur leur histoire. Il a fallu une fois de plus identifier le fantasme de Toute-puissance auquel tout enseignant se frotte à l'occasion quelle que soit sa pratique, y renoncer et admettre que, là comme ailleurs, je pouvais me tromper. Le risque n'était finalement pas démesuré en comparaison à l'attentisme qui était ma seule autre alternative.

\section{Conclusion}

Ce début de recherche ouvre les portes d'une réflexion plus approfondie dans les années à venir mais elle apporte déjà des éléments de conclusion qui peuvent être pris en compte. Globalement il faut noter la nette amélioration des rapports entre élèves et avec l'équipe éducative ainsi que l'entrée dans un rapport au savoir et à l'apprentissage apaisé et moins douloureux, inscrivant l'élève dans une interaction entre lui et le monde, lui permettant d'y agir mais aussi de s'y produire plus librement. Elle apporte par ailleurs un éclairage sur la manière dont se construit aujourd'hui une figure d'élève. Malgré ses limites, l'hétérobiographie peut avoir des vertus émancipatrices et pacificatrices donnant sens à l'utilisation des apports de la recherche biographique dans le cadre de l'éducation formelle, notamment dans certains espaces de prévention et de remédiation aux difficultés des élèves. Si le dispositif n'est pas facilement transférable en tant que tel et vise à produire des connaissances, ses effets immédiats auprès des élèves posent la question de l'espace-temps laissé à la configuration d'une figure d'élève positive dans le cadre scolaire. 


\section{Bibliographie}

BACHELARD D. \& PINEAU G. (Coord.). Le biographique, la réflexivité et les temporalités. Articuler langues cultures et formation. Paris : L'Harmattan, 2009.

BAUTIER E. (Dir.). Apprendre à l'école. Apprendre de l'école. Des risques de construction d'inégalités dès la maternelle. Lyon : Chroniques sociales, 2008.

BLOCH D. Ecole et démocratie. Pour remettre en route l'ascenseur économique et social. Grenoble : PUG, 2010.

BOUMARD P., LAPASSADE G., \& LOBROT M. Le mythe de l'identité. Apologie de la dissociation. Paris : Anthropos, 2006.

BOUTINET J.-P. (Dir. Penser l'accompagnement adulte. Paris : Puf, 2007.

CHARLOT B. Du rapport au savoir. Paris : Anthropos, 2005.

DEWEY J. Démocratie et éducation. Suivi de Expérience et éducation. Paris : Armand Colin, 2011.

DUBET F. Ecole : la question du sens. In : RUANO-BORBALAN, J.-C. (Dir.) Eduquer et former. Les connaissances et les débats en éducation et en formation. Auxerre : Sciences Humaines, 1998, p.405

DELORY-MOMBERGER C. Biographie et éducation. Figures de l'individu-projet. Paris : Anthropos, 2003.

DOLORY-MOMBERGER C. «Biographie, socialisation, formation », L'orientation scolaire et professionnelle [En ligne], 33/4 | 2004, document 4, mis en ligne le 28 septembre 2009, Consulté le 15 août 2011. URL : http://osp.revues.org/index251.html

DELORY-MOMBERGER C. La Condition biographique. Paris : Téraèdre, 2009.

DELORY-MOMBERGER C. Cours sur l'analyse d'entretien, 2010.

DEVELAY M. Donner du sens à l'école. Paris : ESF, 1996.

FABRE, M. Penser la formation. Paris : PUF, 1994.

LAPASSADE G. L'Entrée dans la vie. Essai sur l'inachèvement de l'homme. Paris : Anthropos, 1997. LAPASSADE, G. Microsociologie de la vie scolaire. Paris : Anthropos, 1998.

MAUSS M. Essai sur le don. Paris : PUF, 2007.

MEIRIEU P. Le choix d'éduquer. Paris : ESF, 1997.

NIEWIADOMSKY, C. (2012). Recherche biographique et clinique narrative. Toulouse : Erès, pp. 33 à 41 .

PERRENOUD P. Le Métier d'élève leur appartient. Le travail scolaire entre prescription et activité réelle de l'élève. Educateur, 2005, nº 4, pp. 26-30.

REUTER Y. (Dir.). Une Ecole Freinet. Fonctionnements et effets d'une pédagogie alternative en milieu populaire. Paris : L'Harmattan, 2007.

REUTER Y. L'Analyse du récit. Paris: PUF, 2009.

VARRO G. Temporalité(s) et langage dans l'analyse des entretiens biographiques, 2008. Consulté le 13 avril 2010 sur Temporalités. Revue de sciences sociales et humaines, $n^{\circ} 8$. http://temporalites.revues.org/index123.html.

WULF C. Anthropologie de l'éducation. Paris: L'Harmattan, 1999. 